\title{
Multi Data-Rate Synchronization of 40 GHz Mode-Locked Quantum-Dash Lasers Diodes
}

\author{
Ramón Maldonado-Basilio, Sylwester Latkowski, and Pascal Landais \\ Research Institute for Networks and Communications Engineering \\ School of Electronic Engineering, Dublin City University \\ Corresponding author: landaisp@dcu.ie
}

\begin{abstract}
Beat-tones at 40-GHz from a QDash-ML laser under 10 to $160 \mathrm{~Gb} / \mathrm{s}$ optical injection are analyzed. A FWHM linewidth less than $8 \mathrm{~Hz}$ is obtained regardless of the rate of injected data stream.
\end{abstract}

\begin{abstract}
1. Introduction
Quantum-dash mode-locked (QDash-ML) Fabry-Pérot (FP) laser diodes (LD) have attracted significant interest in optical time- and wavelength-division multiplexing systems due to a variety of their applications. Among the more important are sub-picosecond pulse generation at repetition rates from $40 \mathrm{GHz}$ [1] to $346 \mathrm{GHz}$ [2], frequency multiplication at $270 \mathrm{GHz}$ [3], multi-channel transmission at $10 \mathrm{GHz}$ [4], wavelength tunable transmission at 170 $\mathrm{Gb} / \mathrm{s}$ [5], clock-generation up to $1.2 \mathrm{THz}$ [6], and clock-recovery at $40 \mathrm{~Gb} / \mathrm{s}$ [7]. Regarding the latest mentioned application, the performance of the recovered clock has been assessed either by re-modulating it with the original data-stream and implementing bit-error-rate measurements [7-8], or by analyzing the linewidth of the RF beat-tone signal and retrieving the root mean square timing jitter [9]. In this context, a linewidth analysis of the $40 \mathrm{GHz} \mathrm{RF}-$ beat signal from a QDash-ML FP laser under multi data-rate optical injection is presented. The injected bit rates span from 10 to $160 \mathrm{~Gb} / \mathrm{s}$. Signals consist of non-modulated and modulated trains of 1.6 ps pulses. As a result, it is found that the $40 \mathrm{GHz}$ beat signals exhibit a full-width at half maximum (FWHM) linewidth smaller than $8 \mathrm{~Hz}$ when the laser is under injection, regardless of the injected data-rate. Indeed, such a linewidth is below the resolution of the utilized electrical spectrum analyzer and is smaller than those reported in similar single-section QDash-ML lasers operating under self-injection [10] or in two-section quantum-dot structures operating under passive modelocking [11] conditions. Moreover, harmonic and sub-harmonic synchronization of the QDash-ML laser allows for retrieving a stable $40 \mathrm{GHz}$ clock signal featuring a pulse width of $2 \mathrm{ps}$.
\end{abstract}

\section{Experimental setup}

The QDash-ML laser under investigation is DC-biased, $1 \mathrm{~mm}$ long single section device, without any saturable absorber sections. A brief description of the QDash-based heterostructure, as well as its features and dimensions of the active core have been given in Ref. [1]. The laser under test presents a bias threshold of $18 \mathrm{~mA}$ and a collected average power of $1.6 \mathrm{~mW}$ when operating at $350 \mathrm{~mA}$ and temperature controlled at $25{ }^{\circ} \mathrm{C}$. The QDash-ML laser features more than 40 longitudinal modes with $0.31 \mathrm{~nm}$ inter-modal separation, resulting in a $3 \mathrm{~dB}$ optical bandwidth of $12 \mathrm{~nm}$ centered at $1526 \mathrm{~nm}$. Experimental setup to assess the synchronization performance of the QDash-ML laser is depicted in Fig. 1. A tunable mode-locked laser (TMLL) at $1550 \mathrm{~nm}$ synchronized with an electrical clock from a pattern generator (PPG) is used for generating a train of 1.6 ps pulses at a repetition rate of $10 \mathrm{GHz}$. Back reflections to the TMLL are prevented by connecting an optical isolator (ISO) at its output. An optical time-domain multiplexer (OMUX) is used for bit-rate multiplication, allowing the generation of pulses at repetition rates of 10 , 20, 40, 80 and $160 \mathrm{GHz}$. Optical power at the input and output of the OMUX is enhanced by erbium-doped fiber amplifiers EDFA-1 and EDFA-2. An optical band-pass filter (OBPF-1) is connected at the output of EDFA-2 to

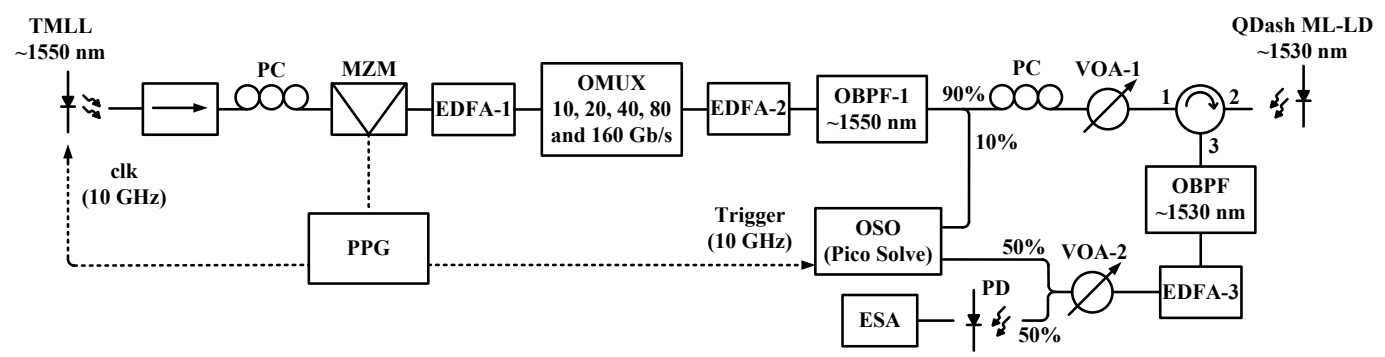

Fig. 1. Experimental setup. Full- and dotted-lines represent optical and electrical links, respectively. 
suppress part of the amplified spontaneous emission of the amplification modules. Pulses at various bit-rates are then injected into the QDash-ML LD through an optical circulator. Recovered clock from the QDash laser is isolated from injected pulses by using an optical filter (OBPF-2) and amplified by EDFA-3. Temporal traces of the recovered clock are analyzed through an optical sampling scope (OSO) with a resolution of $0.8 \mathrm{ps}$ and the beat-tones are detected by a $50 \mathrm{GHz}$ photo-diode (PD) and electrical spectrum analyzer (ESA). As previously stated, two injection conditions of the QDash-ML laser are analyzed in this experiment. The first corresponds to non-modulated train of pulses at various bit-rates, whilst the second corresponds to modulated pulses. In such a case, a MachZehnder modulator (MZM) in combination with the PPG is utilized, generating $\left(2^{31}-1\right)$-long return to zero (RZ) pseudo random binary sequence (PRBS). Polarization controllers (PC) are connected to optimize the operation of the MZM modulator and QDash-ML laser diode. Similarly, variable optical attenuators (VOA-1 and VOA-2) are used to monitor optical power at the laser input and at the optical and electrical detection stages.

\section{Results}

Eye diagrams of the PRBS-pulses injected into the QDash-ML laser are depicted in Fig. 2(a), 2(b) and 2(c). For the sake of simplicity, only pulses at 10,40 and $160 \mathrm{~Gb} / \mathrm{s}$ are shown. As illustrated in Fig. 2(d), synchronization of the QDash-ML laser by any of the analyzed injected data rates allows the retrieval of stable $40 \mathrm{GHz}$ clock pulses exhibiting a pulse width of 2 ps. It is worth mentioning that for all of the injected data rates, sub-harmonic components of the fundamental injected frequency have been minimized by optimizing the operational conditions of the bit-rate multiplier in terms of delay and optical power balance between the time-domain multiplexed data streams, as depicted in Figs. 2(e) to 2(g). Thus, synchronization of the ML laser diode is mainly provided by the fundamental frequency at the given input data rate. This represents a significant feature in the case of injection bitrates above the QDash-ML laser free spectral range due to no spectral component at $40 \mathrm{GHz}$ is necessary for external synchronization, as depicted in Fig. 2(c) and 2(g). Moreover, no eye diagrams are retrieved from the optical sampling scope when the QDash-ML laser is not externally locked. This occurs because of the trigger signal from PPG is not synchronized with the free-running frequency of the laser. In such a case, the $40 \mathrm{GHz}$ beat-tone signal is observed only at the ESA and the QDash-ML LD central frequency and FWHM linewidth resemble to those of the passive mode-locking conditions. In fact, once the QDash-ML laser is synchronized, a shift in the central frequency and a significant reduction in the FWHM-linewidth are observed on the $40 \mathrm{GHz}$ beat-tone signal in respect to the passive mode-locking conditions of the ML laser. As depicted in Fig. 3(a) when injecting $160 \mathrm{~Gb} / \mathrm{s}$ bit sequence, central frequency shifts from 39.8080 to $39.8132 \mathrm{GHz}$, whilst linewidth goes from $70 \mathrm{kHz}$ to less than $8 \mathrm{~Hz}$. A close view of the beat-tones is illustrated in Fig. 3(b) by setting a $600 \mathrm{~Hz}$ span and $10 \mathrm{~Hz}$ bandwidth resolution on the electrical spectrum analyzer. Even at these extreme settings, a linewidth at $-10 \mathrm{~dB}$ of $23 \mathrm{~Hz}$ is retrieved from the ESA, which results in a FWHM inewidth of $7.6 \mathrm{~Hz}$. The same result is obtained by fitting the experimental data with a Lorentzian line-shape. A complete set of recorded data is summarized in Table 1 for all the injection rates from 10 to $160 \mathrm{~Gb} / \mathrm{s}$, considering both modulated and non-modulated input data signals. In all these measurements, operational conditions of the QDash-ML laser are optimized in terms of power (from +6 to $+10 \mathrm{dBm}$ ) and state of polarization of the injected beams, whilst bias current and temperature are fixed to $90 \mathrm{~mA}$ and $25^{\circ} \mathrm{C}$, respectively. A retrieved linewidth below $8 \mathrm{~Hz}$ is obtained regardless of the analyzed data rate of the modulated or non-modulated injected signal. Such a low linewidth is smaller than the $200 \mathrm{~Hz}$ reported in similar single-section $40 \mathrm{GHz}$ QDash-

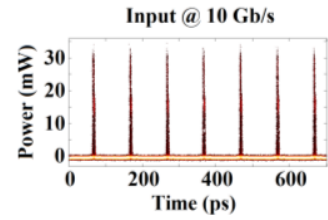

(a)

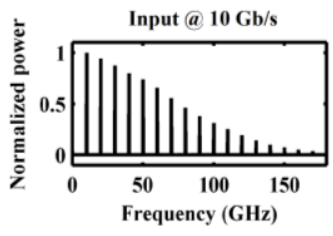

(e)

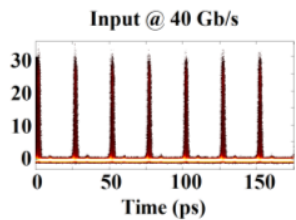

(b)

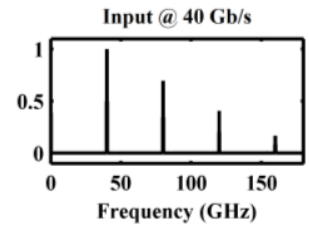

(f)

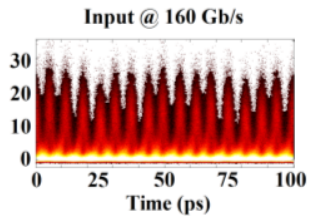

(c)

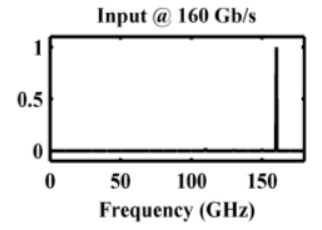

(g)

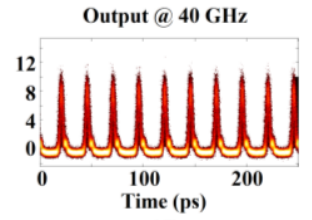

(d)

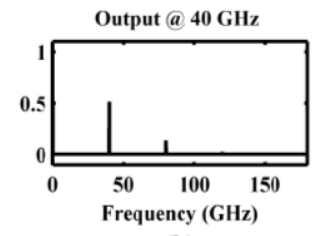

(h)

Fig. 2. (a)-(c): Eye diagram modulated pulses at 10, 40 and $160 \mathrm{~Gb} / \mathrm{s}$ injected to the QDash-MLLD. (d): Retrieved clock pulses at $40 \mathrm{GHz}$ measured at the output of the laser diode. (e)-(h): Spectral content of input and output signals. 

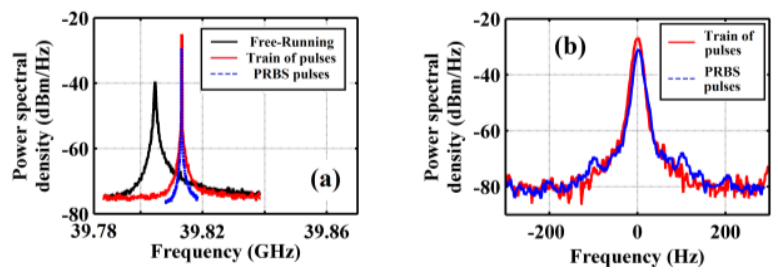

Fig. 3. (a) Beat-tone signal measured at the output of the QDash-ML laser for three different conditions: free running, injection of a nonmodulated train of pulses, and injection of modulated train of pulses, both of them at $160 \mathrm{~Gb} / \mathrm{s}$. (b) Close view of the beat-tones measured when the QDash-ML laser is synchronized with non-modulated and modulated $160 \mathrm{~Gb} / \mathrm{s}$ train of pulses.

Table 1. Summary of the central frequency and FWHM-linewidth associated to the $40 \mathrm{GHz}$ beat-tone signal measured at the output of the QDash-ML for synchronized and passive mode-locking conditions.

\begin{tabular}{c||c|c|c||c|c||c}
\multicolumn{1}{c||}{} & \multicolumn{3}{c||}{ Synchronized Mode Locking } & \multicolumn{2}{c||}{ Passive Mode Locking } & \\
\hline $\begin{array}{c}\text { Bias } \\
(\mathrm{mA})\end{array}$ & Injection rate & $\begin{array}{c}\text { RF-Central } \\
\text { frequency }\end{array}$ & $\begin{array}{c}\text { FWHM } \\
\text { linewidth }\end{array}$ & $\begin{array}{c}\text { RF-Central } \\
\text { frequency }\end{array}$ & $\begin{array}{c}\text { FWHM } \\
\text { linewidth }\end{array}$ & $\begin{array}{c}\text { Locking } \\
\text { range }\end{array}$ \\
\hline 90 & $\begin{array}{c}10,20,40,80 \text { and } \\
160 \mathrm{~Gb} / \mathrm{s}\end{array}$ & $39.8132 \mathrm{GHz}$ & $<8 \mathrm{~Hz}$ & $39.8080 \mathrm{GHz}$ & $70 \mathrm{kHz}$ & $5.2 \mathrm{MHz}$
\end{tabular}

ML lasers operating under self-injection [10], the $500 \mathrm{~Hz}$ reported in two-section $10 \mathrm{GHz}$ quantum-dot structures operating in passive mode-locking [11], and comparable to the $10 \mathrm{~Hz}$ reported in $17 \mathrm{GHz}$ single-section quantumdot electrically mode-locked laser [12].

\section{Conclusions}

Synchronization of a $40 \mathrm{GHz}$ QDash-ML laser for 10 to $160 \mathrm{~Gb} / \mathrm{s}$ non-modulated and modulated pulse sequences has been achieved. For 80 and $160 \mathrm{~Gb} / \mathrm{s}$, no spectral component at $40 \mathrm{GHz}$ was applied for external synchronization. Linewidth of the $40 \mathrm{GHz}$ synchronized signal has been experimentally resolved. A FWHM below $8 \mathrm{~Hz}$ is obtained, improving reported linewidths on similar one-section QDash ML laser or two-section quantum-dot structures.

\section{Acknowledgments}

Authors want to acknowledge Enterprise Ireland under the Programme CFTD/2009/0303.

\section{References}

[1] S. Latkowski, R. Maldonado-Basilio, and P. Landais, "Sub-picosecond pulse generation by 40-GHz passively mode-locked quantum-dash 1mm-long Fabry-Perot laser diode," Opt. Express, 17(21), 19166-19172, 2009.

[2] K. Merghem, A. Akrout, A. Martinez, G. Aubin, A. Ramdame, F. Lelarge, and G.-H. Duan, "Pulse generation at 346 GHz using a passively mode locked quantum-dash-based laser at 1.55 um," Appl. Phys. Lett., 94, 021107, 2009.

[3] M. Xia, C.H. Kwok, M.G. Thompson, R.V. Penty, I.H. White, F.van Dijk, A. Enard, F. Lelarge, and G.-H. Duan, “270GHz, 580fs optical pulse generation from a single-section quantum-dash Fabry-Pérot laser using frequency multiplication,” in CLEO/IQEC 2009, Paper JThE15.

[4] A. Akrout, A. Shen, R. Brenot, F. Van Dijk, O. Legouesigou, F. Pommereau, F. Lelarge, A. Ramdane, and G.-H. Duan, "Separate error-free transmission of eight channels at $10 \mathrm{~Gb} / \mathrm{s}$ using comb generation in a quantum-dash-based mode-locked laser," Photon. Tech. Lett., 21(23), 1746-1748, 2009.

[5] M. Costa e Silva, H. Ramanitra, M. Gay, L. Bramarie, S. Lobo, M. Joindot, J.C. Simon, A. Shen, and G.-H. Duan, "Wavelength tunability assessment of a $170 \mathrm{Gbit} / \mathrm{s}$ transmitter using a quantum dash Fabry Perot mode-locked laser," in ECOC 2009, Paper 6.2.2.

[6] A. Lagrost, M. Costa e Silva, M. Gay, L. Bramerie, S. LaRochelle, P. Besnard, A. Shen, and G.-H. Duan, "Tunable and variable clock generation up to $1.2 \mathrm{THz}$ by filtering an actively mode-locked $42.5 \mathrm{GHz}$ quantum dash Fabry-Perot laser with a reconfigurable filter," in OFC 2010, Paper OWU2.

[7] M. Costa e Silva, A. Lagrost, L. Bramerie, M. Gay, P. Besnard, M. Joindot, J. C. Simon, A. Shen, and G.-H. Duan, "Up to 425 GHz all optical frequency down-conversion clock recovery based on quantum dash Fabry-Perot mode-locked laser," in OFC 2010 , Paper PDPC4.

[8] V. Roncin, A. O’Hare, S. Lobo, E. Jacquette, L. Bramerie, P. Rochard, Q.-T. Le, M. Gay, J.-C. Simon, A. Shen, J. Renaudier, F. Lelarge, and G.-H. Duan, "Multi-data-rate system performance of a 40-GHz all-optical clock recovery based on a quantum-dot Fabry-Pérot laser," Photon. Tech. Lett., 19(19), 1409-1411, 2007.

[9] X. Tang, J.C. Cartledge, A. Shen, A. Akrout, and G.-H. Duan, "Low-timing-jitter all-optical clock recovery for 40 Gbits/s RZ-DPSK and NRZ-DPSK signals using a passively mode-locked quantum-dot Fabry-Perot semiconductor laser,” Opt. Lett., 34(7), 899-901, 2009.

[10] A. Akrout, A. Shen, A. Enard, G.-H. Duan, F. Lelarge, and A. Ramdane, "Low phase noise all-optical oscillator using quantum dash mode locked laser," Electron. Lett., 46(1), 73-74, 2010.

[11] G. Carpintero, M.G. Thompson, R.V. Penty, and I.H. White, "Sub-kHz RF electrical linewidth from a 10 GHz passively mode-locked quantum-dot laser diode," in CLOE 2009, Paper CMK2.

[12] A. Shen, F. van Dijk, J. Renaudier, G.-H. Duan, F. Lelarge, F. Pommereau, F. Poingt, L. Le. Gouezigou, and O. Le Gouezigou, “Active mode-locking of quantum-dot Fabry-Perot laser diode," in Semiconductor Laser Conference 2006, Paper ThB6, $153-154$. 